\title{
Varicella Pneumonia in Immunocompetent Adults: Report of Two Cases, with Emphasis on High-Resolution Computed Tomography Findings
}

Emerson L. Gasparetto ${ }^{\mathbf{1}}$, Danny Warszawiak ${ }^{\mathbf{1}}$, Priscilla Tazoniero $^{1}$, Dante L. Escuissato ${ }^{1}$ and Edson Marchiori ${ }^{2}$
Radiology Department of the University of Paraná ${ }^{1}$; Curitiba, PR; Radiology Department of the University of Rio de Janeiro ${ }^{2}$; Rio de Janeiro, RJ, Brazil

\begin{abstract}
We report two cases of varicella pneumonia in immunocompetent patients, with emphasis on high-resolution computer tomography manifestations. The predominant findings consisted of multiple bilateral nodules, ranging from $1-10 \mathrm{~mm}$ in diameter, with or without a surrounding halo of ground-glass attenuation. Other findings include ground-glass opacities, focal areas of consolidation and small pleural effusions.

Key Words: Lung diseases, lung infection, computed tomography, high-resolution computed tomography.
\end{abstract}

Varicella is a common benign childhood illness caused by varicella-zoster virus, typically associated with fever and a characteristic exanthematous vesicular rash [1-3]. The clinical presentation in adults is more severe and more commonly associated with complications, particularly varicella pneumonia [1-5]. Varicella pneumonia has been reported in $5-50 \%$ of adults with chickenpox [1, 2,5]. Most cases occur in immunocompromised adults, in whom it is associated with considerable morbidity and mortality $[1,5]$. The computer tomography (CT) findings have been described in a small number of cases as consisting of multiple well- or ill-defined 1-10 mm diameter nodules diffusely distributed throughout the lungs, with or without a halo of ground-grass attenuation [5-8]. We report two cases of varicella pneumonia in immunocompetent adults, emphasizing the highresolution CT findings.

Received on 07 March 2004; revised 0 June 2005.

Address for correspondence: Dr. Emerson L. Gasparetto. Av. Silva Jardim 296 ap 502. Zip code: 80230-000 Curitiba, PR. Email: gasparetto@hotmail.com

The Brazilian Journal of Infectious Diseases 2005;9(3):262-265 (C) 2005 by The Brazilian Journal of Infectious Diseases and Contexto Publishing. All rights reserved.

\section{Case Reports}

$\underline{\text { Case } 1}$

A 32-year-old female presented with a two-day history of dyspnea, pleuritic pain and hemoptysis. She had an exanthematous vesicular rash and fever that had started a week prior to the respiratory symptoms. She was a cigarette smoker (20 cigarettes per day for 10 years) and her two sons had recently had chickenpox. Physical examination revealed tachypnea, widespread crepitations and rhonchi, enlargement of the spleen and liver, inferior limb edema and multiple vesicles, pustules and crusty skin lesions.

Chest radiography revealed cardiomegaly, bilateral poorly-defined nodular opacities and a small pleural effusion. High-resolution CT showed multiple 1-10mm diameter nodules, randomly distributed throughout both lungs (Figure 1). Most of the nodules had well-defined margins, and many were surrounded by a halo of ground glass attenuation. Bilateral ground glass opacities, small foci of consolidation in the lower lobes, and a small left-side pleural effusion, were also noted. Sputum examination (bacterioscopy and culture) did not show any evidence of bacterial infection. Pleural effusion examination showed a predominance of mononuclear 
cells and no bacterial growth on culture. Electrocardiography revealed a left branch block.

A diagnosis of varicella pneumonia and carditis was made based on the characteristic polymorphic skin rash and the history of contact with chickenpox; the patient was started on intravenous acyclovir. Follow-up highresolution $\mathrm{CT}$, seven days after the start of therapy, demonstrated a decrease in the number and size of pulmonary nodules and a persistent left-side pleural effusion. The chest radiograph performed 14 days later showed complete resolution of the pulmonary lesions and persistent cardiomegaly.

\section{Case 2}

A 30-year-old female presented a five-day history of cough, fever, dyspnea and cyanosis. She had an exanthematous vesicular rash that had started three days prior to the respiratory symptoms. She was a cigarette smoker (15 cigarettes per day for 8 years) and her daughter had recently had chickenpox. Physical examination showed tachypnea, cyanosis, fever, and widespread rhonchi. Skin examination revealed a characteristic polymorphic rash, with vesicles, pustules and crusty lesions.

Chest radiography showed multiple small nodules. A high-resolution CT revealed numerous ill-defined 1$5 \mathrm{~mm}$ centrilobular nodules, randomly distributed in both lungs. Coalescence and surrounding ground-glass attenuation halos were also observed in some nodules. The diagnosis of varicella pneumonia was made based on the presence of a typical skin rash, pulmonary symptoms and contact with a child with chickenpox; the patient was started on intravenous acyclovir. She had a complete recovery after two weeks, with complete resolution of the lung lesions, based on a chest radiograph.

\section{Discussion}

Varicella pneumonia usually occurs 1 to 6 days after the onset of a varicella-zoster infection. The clinical manifestations include cough, dyspnea, tachypnea, fever, cyanosis, and sometimes pleuritic chest pain or haemoptysis [1,5]. Most adult patients with varicella pneumonia are immunocompromised, most commonly in a setting of leukemia, lymphoma and corticosteroid therapy [5]. Other risk factors include pregnancy, smoking, old age and chronic obstructive pulmonary disease [2-5]. Mortality is $10-30 \%$ in the general population, approximately $40 \%$ in pregnancy, and greater than $50 \%$ in immunocompromised patients [1]. Our patients presented with typical respiratory symptoms after contact with individuals with varicella. Both patients were healthy adults, with smoking as the only potential risk factor.

Histological features of pneumonia caused by the varicella-zoster virus consist of an interstitial mononuclear inflammatory infiltrate, associated with intraalveolar proteinaceous exudate, hyaline membrane formation, and type II cell hyperplasia. After recovery from the initial disease, spherical nodules are seen, randomly distributed in the lung parenchyma. Histologically, the nodules are composed of an outer, often lamellated fibrous capsule, frequently enclosing areas of hyalinized collagen or necrotic tissue. The nodules frequently calcify $[5,7]$.

The characteristic radiological manifestations consist of multiple bilateral 5-10 mm, ill-defined nodules, which may be confluent [1,3-7,9]. Occasionally, hilar lymphadenopathy, reticular opacities and pleural effusion may occur [1,5,7]. Picken et al. [6] described the high-resolution CT findings of varicella pneumonia in a immunocompromised patient who received chemotherapy for treatment of a malignant testicular germ cell tumor. They observed multiple bilateral soft tissue nodules, measuring 5-10 $\mathrm{mm}$ in diameter. These nodules were visible on high-resolution CT, but not with plain chest radiography, raising the possibility that varicella pneumonia occurs more frequently than presently suspected.

Kim et al. [5] presented high-resolution CT findings for three immunocompetent patients with varicella pneumonia. The abnormalities included 1-10 mm diameter well- and ill-defined nodules, diffusely dispersed throughout both lungs; these nodules had a surrounding halo of ground-glass attenuation, patchy 
Figure 1. 32-year-old woman with varicella pneumonia.1A. High resolution computerized tomography (CT) at the level of the bronchus intermedius demonstrates multiple bilateral nodules. Most nodules have well defined margins, and many are surrounded by a halo of ground glass attenuation. 1B. High resolution CT at the level of the inferior pulmonary veins demonstrates bilateral small nodules, ground glass opacities, and focal areas of consolidation.
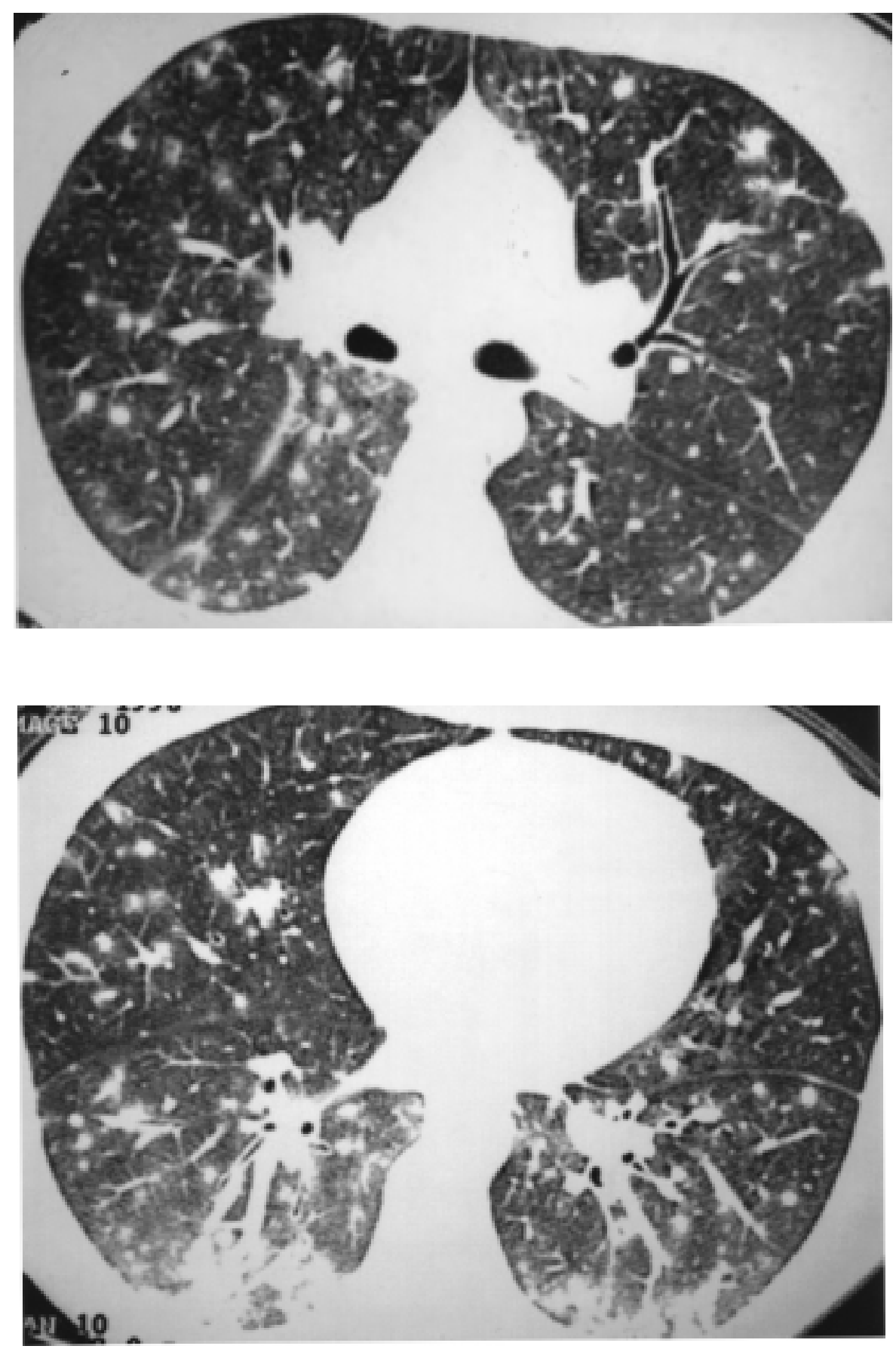
ground-glass attenuation, and coalescence of lesions. The nodules were randomly distributed in the secondary pulmonary lobules and showed no predilection for any particular area of the lung. None of the patients had pleural effusion or lymphadenopathy. Most of the highresolution CT findings in our patients were similar to those reported in this paper, consisting of numerous well- or ill-defined 1-10 mm centrilobular nodules, some of then with ground-glass halos, randomly distributed in both lungs. However, our cases had ground-glass opacities, focal areas of consolidation and small pleural effusions, which were not previously reported in patients with varicella pneumonia.

In conclusion, the high-resolution CT manifestations of varicella pneumonia consist of multiple bilateral nodules, ranging from 1-10 $\mathrm{mm}$ in diameter with or without a surrounding halo of ground-glass attenuation. Other findings include ground-glass opacities, focal areas of consolidation and small pleural effusions.

\section{References}

1. Gregorakos L., Myrianthefs P., Markou N., et al. Severity of illness and outcome in adult patients with primary varicella pneumonia. Respiration 2002;69:330-4.

2. Jones A.M., Thomas N., Wilkins E.G..L. Outcome of varicella pneumonitis in immunocompetent adults requiring treatment in a high dependency unit. J Infect 2001;43:135-9.

3. Pugh R.N., Omar R.I., Hossain M.M. Varicella infection and pneumonia among adults. Int J Infect Dis 1998;2:20510.

4. Mohsen A.H., Peck R.J., Mason Z., et al. Lung function tests and risk factors for pneumonia in adults with chickenpox. Thorax 2001;56:796-9.

5. Kim J.S., Ryu C.W., Lee S.I., et al. High-resolution CT findings of varicella-zoster pneumonia. Am J Roentgenol 1999; 172:113-6.

6. Picken G., Booth A.J., Williams M.V. Case report: the pulmonary lesions of chickenpox pneumonia - revisited. Br J Radiol 1994;67:659-60.

7. Kim E.A., Lee K.S., Primack S.L., et al. Viral pneumonia in adults: radiologic and pathologic findings. RadioGraphics 2002;22:S137-49.

8. Nakamura M., Kanazawa M., Yamaguchi K., et al. Pneumonia caused by varicella-zoster virus in a patient with rheumatoid arthritis. Jpn J Thorac Dis 1996;67:610-5.
9. Eskenazi A.E., Campbell A.B. Radiological case of the month. Varicella mimicking pulmonary metastasis in a patient with rabdomyosarcoma. Arch Pediatr Adolesc Med 1997;151:1051-2. 